\title{
Auxílio da tomografia computadorizada no planejamento pré-operatório de pacientes portadores de escoliose idiopática do adolescente
}

\author{
Computed tomography aid in preoperative planning of patients with \\ adolescent idiopathic scoliosis
}

\author{
Auxilio de la tomografía computarizada en el plan preoperatorio de \\ pacientes portadores de escoliosis idiopática del adolescente
}

\author{
Rodrigo Rezende ${ }^{1}$ \\ Igor Cardoso Machado² \\ Diogo Miranda Barbosa ${ }^{3}$ \\ Priscila Rossi de Batista ${ }^{4}$
}

\section{RESUMO}

A escoliose idiopática do adolescente (EIA) é uma doença diagnosticada no início da puberdade. Ela apresenta etiologia indefinida e caracteriza-se pelo desvio lateral da coluna vertebral maior que $10^{\circ}$, associado à rotação do corpo vertebral. O uso de instrumentação de terceira geração produziu um melhor resultado na correção da curva do que as técnicas anteriores. Porém, ainda existem dificuldades técnicas no momento da passagem e da escolha dos parafusos pediculares, devido à angulação e ao diâmetro dos pedículos vertebrais. Apesar de apresentar vantagens, essa técnica não é isenta de riscos e complicações, necessitando de uma maior curva de aprendizado pelo médico-cirurgião, bem como de um adequado planejamento pré-operatório. Até o momento, não há na literatura relato sobre a utilização da tomografia computadorizada no auxílio do planejamento cirúrgico

\section{ABSTRACT}

The adolescent idiopathic scoliosis is a diagnosed disease in the beginning of puberty, with unknown etiology, characterized by lateral deviation of the spine (above $10^{\circ}$ ), which is related to vertebral rotation. The form of surgical treatment by spinal fixation with pedicle screws showed better results when compared to other fastening systems, but there are still technical difficulties at the time of passage and the choice of pedicle screws because of vertebral pedicles angle and diameter. Despite its advantages, this technique presents risks and complications, requiring a greater learning curve for the surgeon, as well as an adequate preoperative planning. So far, there is no literature report about the use of computed tomography as an aid to surgical planning of patients with scoliosis. In this

\section{RESUMEN}

La escoliosis idiopática en el adolescente (EIA) es una enfermedad diagnosticada en el inicio de la pubertad. Esta presenta una etiología indefinida y es caracterizada por el desvío lateral de la columna vertebral por encima de los diez grados asociados a la rotación del cuerpo vertebral. El uso de instrumentación de tercera generación produjo un mejor resultado en la corrección de la curva comparado con las técnicas anteriores. Sin embargo, existen dificultades técnicas en el momento del paso $y$ de escoger los tornillos pediculares debido a la angulación y al diámetro de los pedículos vertebrales. A pesar de presentar ventajas, esta técnica no está libre de riesgos y complicaciones, necesitando de una mayor curva de aprendizaje por el médico cirujano, así como también de un adecuado plan preoperatorio. Hasta el momento, no hay en la literatura un relato sobre la utilización de la tomografía computarizada en el

\footnotetext{
Trabalho realizado no Hospital Santa Casa de Misericórdia de Vitória - Vitória (ES), Brasil.

'Mestre; Ortopedista e traumatologista do Hospital Santa Casa de Misericórdia de Vitória - Vitória (ES) e do Vila Velha Hospital - Vila Velha (ES), Brasil. ${ }^{2}$ Ortopedista e traumatologista do Hospital Santa Casa de Misericórdia de Vitória - Vitória (ES) e do Hospital Meridional - Cariacica (ES), Brasil.

${ }^{3}$ Radiologista do Centro de Diagnóstico por Imagem - CDI - Vitória (ES), Brasil.

${ }^{4}$ Pós-graduanda (Doutorado) em Ciências Fisiológicas da Universidade Federal do Espírito Santo - UFES - Vitória (ES), Brasil.
} 
de pacientes com escoliose. Diante do exposto, objetivamos, no presente estudo, descrever uma técnica de planejamento pré-operatório, com o auxílio da tomografia computadorizada para a fixação da coluna em pacientes com escoliose idiopática do adolescente, especificando a angulação e o diâmetro dos pedículos vertebrais, o que poderá auxiliar o médico-cirurgião no momento da fixação.

DESCRITORES: Tomografia computadorizada por raios X; Coluna vertebral; Escoliose; Dispositivos de fixação ortopédica

light, the present study aims to describe a preoperative planning technique with the aid of computed tomography for spinal fixation in patients with Adolescent Idiopathic Scoliosis, specifying the angle and diameter of the vertebral pedicles, which may help the surgeon in the fixation.

KEYWORDS: Tomography, X-ray computed; Spine; Scoliosis; Orthopedic fixation devices auxilio quirúrgico de los pacientes con escoliosis. Según lo expuesto, proponemos, en el presente estudio, describir una técnica para el plan preoperatorio con el auxilio de la tomografia computarizada para la fijación de la columna en pacientes con escoliosis idiopática del adolescente, especificando la angulación y el diámetro de los pedículos vertebrales, lo que podrá auxiliar al médico cirujano en el momento de la fijación.

DESCRIPTORES: Tomografía computadorizada por rayos $\mathrm{X}$; Columna vertebral; Escoliosis; Dispositivos de fijación ortopédica

\section{INTRODUÇÃO}

A escoliose idiopática do adolescente (EIA) é caracterizada por uma curvatura lateral da coluna vertebral, acima de $10^{\circ}$, associada à rotação do corpo vertebral, sendo que o início da deformidade ocorre durante a puberdade e sua etiologia permanece indefinida. A evolução cursa de forma assintomática, vindo a ser diagnosticada mais tarde, produzindo problemas físicos e sociais ${ }^{1,2}$. Estima-se que de 2 a $3 \%$ da população apresente algum grau de escoliose, sendo o sexo feminino acometido três vezes mais que o masculino ${ }^{3}$.

A detecção precoce dessa deformidade vertebral interfere na forma de tratamento e na evolução dos pacientes. Muitos deles, quando detectados de modo precoce, são submetidos a tratamento apenas por meio da observação periódica de sua deformidade, enquanto outros deverão utilizar coletes corretivos. Em alguns casos em que a deformidade atinge valores maiores que $40^{\circ}$, o tratamento cirúrgico é imperativo, pois pode haver progressão da deformidade, levando a comprometimento do desenvolvimento físico dos pacientes e a alterações cardiorrespiratórias.

Durante as duas últimas décadas, ocorreu um grande avanço no tratamento cirúrgico dessas deformidades, deixando, muitas vezes, de se realizar artrodese in situ associada à imobilização gessada por longos períodos de tempo - para serem realizadas artrodeses com utilização de parafusos pediculares ${ }^{4-7}$, apresentando maior poder de correção da deformidade, além de não ser necessária a utilização de imobilização gessada.

Apesar dos avanços relacionados à utilização dos parafusos pediculares, essa técnica não está isenta de complicações, sendo a mais frequente a não fixação do parafuso em local apropriado, podendo invadir a cortical medial e causar lesão neurológica, ou invadir a cortical lateral e causar complicações pulmonares, como pneumotórax ${ }^{7,8}$.
Essas complicações ocorridas durante a passagem dos parafusos pediculares são ainda maiores nas deformidades vertebrais, devido à modificação dos pontos anatômicos causada pela rotação vertebral ${ }^{9,10}$.

Diante do exposto, o objetivo do nosso trabalho foi descrever uma técnica, por meio de tomografia computadorizada (TC), para análise pré-operatória da anatomia da coluna vertebral em pacientes com deformidades vertebrais causadas pela EIA, relatando a angulação e o diâmetro dos pedículos vertebrais.

\section{DESCRIÇÃO TÉCNICA}

Padronizamos o posicionamento dos pacientes com deformidades vertebrais em decúbito dorsal, mantendo o ápice da giba costal apoiada sobre a mesa de exames e a concavidade da curva apoiada sobre coxins, com a finalidade de evitar angulação no plano coronal. Desta forma, todos os pacientes ficaram com o tronco paralelo ao solo, simulando o posicionamento na mesa cirúrgica operatória.

Foram obtidas imagens de todos os pacientes, da terceira vértebra torácica (D3) até a segunda vértebra lombar (L2), local esperado para instrumentação cirúrgica. Após a revisão das imagens adquiridas no plano axial, as mesmas foram analisadas e trabalhadas por meio do software visualizador de imagens DICOM Osirix ${ }^{\circledR}$ (Machintosh), disponibilizado de modo gratuito na internet, compatível com computadores pessoais, não sendo necessária a utilização de workstation.

Os exames foram realizados em aparelho de TC helicoidal High Speed ${ }^{\circledR}$ (GE Healthcare, USA). Imagens axiais foram obtidas utilizando-se espessura de corte de $3 \mathrm{~mm}$, sem espaçamento entre as mesmas, com pitch de 1, resolução de 512 x 512 pixels, e então foram trabalhadas com o software visualizador de imagens DICOM Osirix ${ }^{\circledR}$ (Machintosh). 
O estudo tomográfico dos pacientes com escoliose vertebral baseou-se na análise da angulação e na mensuração do diâmetro dos pedículos das vértebras de D3 até L2. Durante a mensuração do diâmetro dos pedículos, as imagens foram adquiridas no plano axial e reconstruídas utilizando-se a ferramenta MPR ${ }^{\circledR}$ "multiplanar reconstruction tornando possível a criação de um plano axial oblíquo e demonstrando ambos os pedículos na mesma imagem e o real diâmetro dos mesmos, sem a interferência da angulação vertebral nos planos coronal e axial. Após aquisição da imagem no plano axial oblíquo, foi realizada a mensuração do diâmetro dos pedículos (DP), que é a medida do menor diâmetro mediolateral, perpendicular ao eixo pedicular, incluindo as corticais ósseas. Os parâmetros lineares foram medidos em milímetros, utilizando-se uma casa decimal após a vírgula (Figura 1).

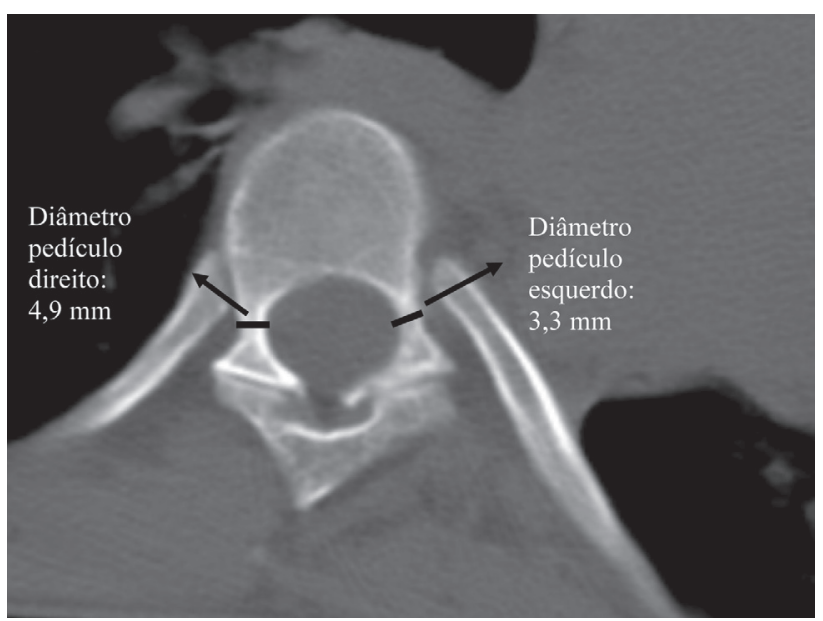

Figura 1

Mensuração do diâmetro pedicular (DP).

Para o cálculo da angulação dos pedículos (AP), traçou-se uma linha no plano horizontal, paralela ao plano da mesa de estudo da TC e, consequentemente, paralela ao solo. Essa linha corresponde ao plano visualizado pelo cirurgião, visto que o paciente se encontra em decúbito ventral no momento da cirurgia. - e uma outra outra linha, paralela ao eixo de cada pedículo. O cálculo da angulação pedicular foi obtido entre o cruzamento da linha no plano horizontal e a linha do eixo pedicular (Figura 2).

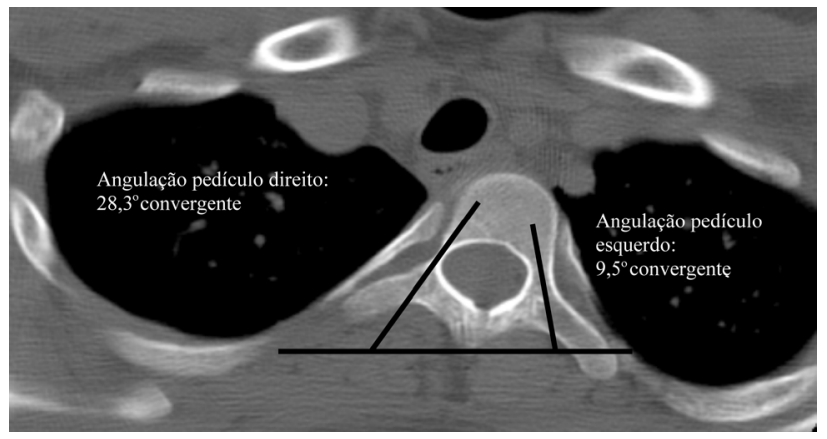

Figura 2

Mensuração da angulação da rotação pedicular (AP).
A esse ângulo é adicionado um sinal de positivo $(+)$, quando o eixo do pedículo converge para a linha média do corpo do paciente, e negativo (-), quando o eixo do pedículo diverge da linha média do corpo do paciente.

Após o cálculo do DP e da AP de todas as vértebras incluídas no estudo (D3 a L2), foi então preparado um esquema ilustrativo, incluindo todas as medidas realizadas, separadas por suas respectivas vértebras (Figura 3).

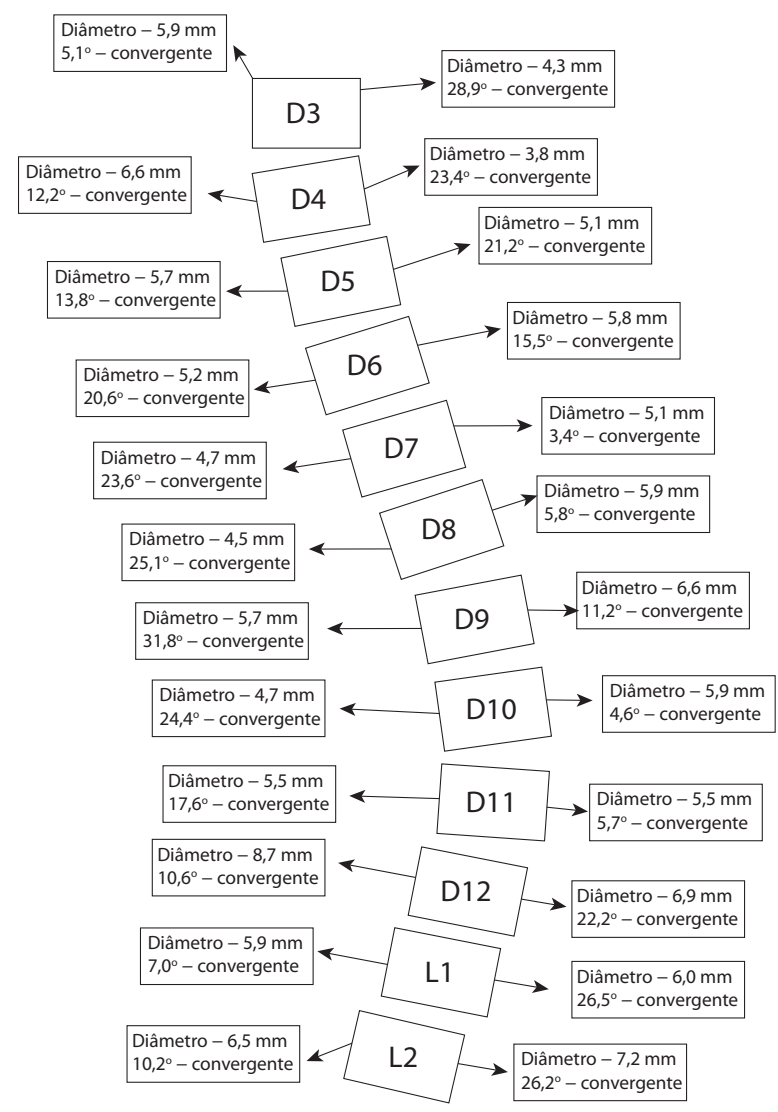

ESQUERDA

DIREITA

Figura 3

Esquema de todas as medidas realizadas da angulação dos pedículos (AP) e do diâmetro dos pedículos (DP) das vértebras estudadas (D3 a L2).

\section{DISCUSSÃO}

O estado atual do tratamento cirúrgico das deformidades vertebrais deve-se ao desenvolvimento e à difusão da técnica de fixação da coluna vertebral com a utilização dos parafusos pediculares ${ }^{4-7}$. Esse tipo de fixação apresenta vantagens em relação às fixações com Harri-Luque, ganchos ou fixações híbridas, utilizando-se parafusos e ganchos por apresentarem maior poder de correção e menor nível de vértebras artrodesadas. No entanto, essa técnica necessita de uma curva de aprendizado pelo médico-cirurgião mais demorada, relacionada a uma maior taxa de complicações, dentre elas, o posicionamento inadequado dos parafusos nos pedículos ${ }^{4,5,7}$.

A motivação para a realização deste trabalho foi divulgar uma técnica de planejamento pré-operatório, por 
meio de TC para o tratamento cirúrgico das deformidades vertebrais relacionadas aos pacientes portadores de EIA, diminuindo as complicações inerentes a esse tratamento. Desta forma, além da diminuição do tempo operatório e do sangramento, o cirurgião pode escolher com mais segurança o diâmetro dos parafusos a serem utilizados.

Vários autores já descreveram a utilização de métodos de imagem na análise pré-operatória de pacientes com EIA ${ }^{6,9-15}$, enfatizando o cálculo do diâmetro dos pedículos ou a avaliação da rotação vertebral. Ao que nos consta, este é o primeiro trabalho em que a análise se baseia na angulação independente de cada pedículo vertebral, visto que os trabalhos por nós revisados mensuram a angulação vertebral $^{11,13-15}$, não levando em consideração a deformidade da vértebra e de seus constituintes, tais como pedículos, lâminas, processo espinhoso e processo transverso ${ }^{9,10}$.

Com a análise do diâmetro do pedículo vertebral, é possível estimar melhor o tamanho do parafuso pedicular a ser utilizado, evitando, desta forma, complicações como a quebra da cortical medial ou lateral dos pedículos, por meio da utilização de parafusos de diâmetro adequado. Outro ponto a ser discutido é a utilização de parafusos pediculares para a fixação vertebral em pedículos com diâmetro muito pequeno, visto que foram encontrados, em nossos dados, pedículos com diâmetros inferiores a $2 \mathrm{~mm}$.

A avaliação pré-operatória com TC também permitiu analisar a angulação da rotação dos pedículos vertebrais em relação ao eixo horizontal, simulando o posicionamento do paciente na mesa operatória. Essa análise fornece uma estimativa da posição de entrada dos parafusos pediculares, diminuindo os riscos de complicações neurológicas e pulmonares, como paraplegia e pneumotórax.

Existem outros métodos mais sofisticados para a análise da angulação da rotação vertebral, como a navegação guiada por imagem com utilização de $\mathrm{TC}^{16}$, em que há necessidade de um longo tempo intraoperatório para sua realização, aumentando os riscos anestésicos e a taxa de infecção operatória, além de requerer um equipamento de alto custo que poucos hospitais apresentam condições para sua aquisição.

A análise do diâmetro e angulação pedicular por meio de TC é de fácil utilização e interpretação pelo médicocirurgião, o qual deverá apenas localizar o melhor ponto de entrada do parafuso pedicular sob visão do intensificador de imagens, sendo que a inclinação da mão para a passagem do parafuso deverá ser estabelecida pela análise prévia da TC.

Esse método pode auxiliar, principalmente, cirurgiões com menor prática no tratamento cirúrgico das deformidades vertebrais, reduzindo a curva de aprendizado e dando maior segurança para o ato operatório.

\section{CONCLUSÃO}

A avaliação pré-operatória de pacientes com EIA por meio de TC visa fornecer ao cirurgião medidas precisas do diâmetro dos pedículos e de suas angulações em relação ao plano horizontal, podendo se tornar uma importante ferramenta no sentido de reduzir o tempo intraoperatório e as complicações do posicionamento inadequado dos parafusos pediculares.

\section{REFERÊNCIAS}

1. Lonstein JE. Screening for spinal deformities in Minnesota schools. Clin Orthop Relat Res. 1977;(126):33-42.

2. Yawn BP, Yawn RA, Hodge D, Kurland M, Shaughnessy WJ, Ilstrup $\mathrm{D}$, et al. A population-based study of school scoliosis screening. JAMA. 1999;282(15):1427-32.

3. Velezis MJ, Sturm PF, Cobey J. Scoliosis screening revisited: findings from the District of Columbia. J Pediatr Orthop. 2002;22(6):788-91

4. Kim YJ, Lenke LG, Bridwell KH, Cho YS, Riew KD. Free hand pedicle screw placement in the thoracic spine: is it safe? Spine (Phila Pa 1976). 2004;29(3):333-42.

5. Kim YW, Lenke LG, Kim YJ, Bridwell KH, Kim YB, Watanabe $\mathrm{K}$, et al. Free-hand pedicle screw placement during revision spinal surgery: analysis of 552 screws. Spine (Phila Pa 1976). 2008;33(10):1141-8.
6. Lee CS, Kim MJ, Ahn YJ, Kim YT, Jeong KI, Lee DH. Thoracic pedicle screw insertion in scoliosis using posteroanterior $\mathrm{C}$-arm rotation method. J Spinal Disord Tech. 2007;20(1):66-71.

7. Kim YJ, Lenke LG. Thoracic pedicle screw placement: free-hand technique. Neurol India. 2005;53(4):512-9.

8. Di Silvestre M, Parisini P, Lolli F, Bakaloudis G. Complications of thoracic pedicle screws in scoliosis treatment. Spine (Phila Pa 1976). 2007;32(15):1655-61.

9. Kotwicki T, Napiontek M. Intravertebral deformation in idiopathic scoliosis: a transverse plane computer tomographic study. J Pediatr Orthop. 2008;28(2):225-9.

10.O'Brien MF, Lenke LG, Mardjetko S, Lowe TG, Kong Y, Eck K, et al. Pedicle morphology in thoracic adolescent idiopathic scoliosis: is pedicle fixation an anatomically viable technique? Spine (Phila Pa 1976). 2000;25(18):2285-93.
11. Kouwenhoven JW, Vincken KL, Bartels LW, Meij BP, Oner FC, Castelein RM. Analysis of preexistent vertebral rotation in the normal quadruped spine. Spine (Phila $\mathrm{Pa}$ 1976). 2006;31(20):E754-8.

12.Kim YJ, Lenke LG, Cheh G, Riew KD. Evaluation of pedicle screw placement in the deformed spine using intraoperative plain radiographs: a comparison with computerized tomography. Spine (Phila Pa 1976). 2005;30(18):2084-8.

13. Yazici M, Acaroglu ER, Alanay A, Deviren V, Cila A, Surat A. Measurement of vertebral rotation in standing versus supine position in adolescent idiopathic scoliosis. J Pediatr Orthop. 2001;21(2):252-6.

14.Chi WM, Cheng CW, Yeh WC, Chuang SC, Chang TS, Chen JH. Vertebral axial rotation measurement method. Comput Methods Programs Biomed. 2006;81(1):8-17. 
15.Lam GC, Hill DL, Le LH, Raso

JV, Lou EH. Vertebral rotation measurement: a summary and comparison of common radiographic and CT methods. Scoliosis. 2008;3:16.
16.Kim KD, Patrick Johnson J, Bloch BS O, Masciopinto JE. Computer-assisted thoracic pedicle screw placement: an in vitro feasibility study. Spine (Phila Pa 1976). 2001;26(4):360-4.

\section{Correspondência}

Rodrigo Rezende

Rua Desembargador Augusto Botelho, 209/801 - Praia da Costa

CEP: 29101-110 - Vila Velha (ES), Brasil

Tel.: (27) 2127-8990

E-mail: grupodecoluna@santacasavitoria.org

\section{ERRATA}

No artigo:"Avaliação clínica e funcional no pré-operatório de doenças degenerativas da coluna vertebral", na página pagina 248 onde se lê: “O coeficiente $r$ de correlação de Spearman entre a HAD-D e o IDB foi $\mathbf{0 , 6 6 3}$ (p < 0,001)." leia-se O coeficiente $r$ de cor-relação de Spearman entre a HAD-D e o IDB foi $\mathbf{0 , 7 1 4}(\mathrm{p}<0,001)$. 